\title{
Research Article \\ Electromagnetic Topology Analysis to Coupling Wires Enclosed in Cavities with Apertures
}

\author{
Ying Li, Jianshu Luo, Guyan Ni, and Jiyuan Shi \\ Department of Mathematics and System Science, College of Science, National University of \\ Defense Technology, Changsha, Hunan 410073, China \\ Correspondence should be addressed to Ying Li, liying801@163.com
}

Received 17 February 2010; Revised 8 July 2010; Accepted 1 September 2010

Academic Editor: Mohammad Younis

Copyright ( $) 2010$ Ying Li et al. This is an open access article distributed under the Creative Commons Attribution License, which permits unrestricted use, distribution, and reproduction in any medium, provided the original work is properly cited.

\begin{abstract}
We use both electromagnetic topology (EMT) and the Baum-Liu-Tesche (BLT) equation to analyze a cavity model with an aperture. More precisely, we combine the aperture coupling theory and EMT to study the issues of the electromagnetic field penetration through apertures into a cavity and the coupling to a two-wire transmission line in it. We employ the equivalence principle to establish the equivalent source on the aperture. Then, we obtain the semi analytic solutions of the load response of the two-wire line in the cavity based on the Baum-Liu-Tesche (BLT) equation. In addition, based on the Agrawal model, we give the coupling current distribution at two loads for a two-wire line in the cavity. Finally, we present some numerical results to demonstrate the semi-analytic approach of this paper. In fact, these numerical results on the electric field shielding (EFS) of a rectangular cavity with an aperture agree well with the experimental results in the literature. Furthermore, for a two-wire line in the cavity with an aperture the induced current peaks at loads are observed in the frequency range, some of which are associated with the resonance of the aperture, and others correspond to the resonant frequencies of the cavity.
\end{abstract}

\section{Introduction}

Electromagnetic interference and compatibility have become an integral part in the design and analysis of electronic components and systems. Through an aperture, an external electromagnetic pulse (EMP) may couple to an electronic system in the cavity and induce the transient current and voltage. It is well known that both the transient current and voltage may damage some critical components in the system. Therefore, it is very important to study these interaction processes (see [1-10]).

Electromagnetic interaction problems on very large and complex system, such as an aircraft, can be simulated through using codes based on EMT. An important ingredient of topological analysis is the determination of a mechanism to represent external-internal coupling through a small aperture and the subsequent propagation process. By combining the 


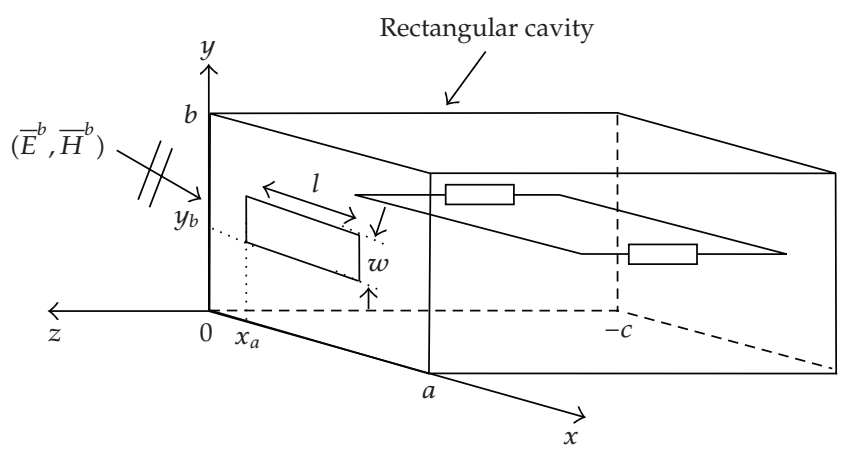

Figure 1: Cavity geometry enclosing a two-wire transmission line.

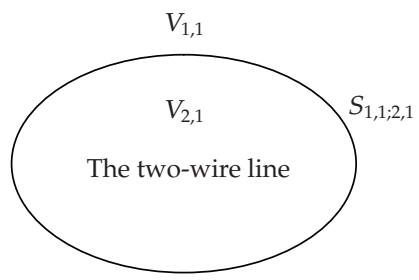

Figure 2: Topological shielding diagram corresponding to Figure 1.

methodologies proposed in [1], one can create an equivalent source provided that the source of aperture radiation is an imaginary transmission line (see $[2,3])$. Such an equivalent source will relate the electromagnetic coupling at the exterior surface and the transfer function through free space generated by it.

In this paper, we apply EMT to analyze the problem of the EM interaction in an electronic system which contains a cavity, an aperture, and a two-wire transmission line. First, based on the equivalence principle, we obtain an equivalent source for the aperture. The idea is to use a semianalytical approach based on the Modal Green Function and the method of moment (MoM). A similar result has been independently shown in [6]. Secondly, we employ the BLT equation to compute the load response of a two-wire transmission line in the cavity with an aperture. Finally, we present several numerical examples to confirm the validity of our results.

\section{Application of Electromagnetic Topology}

The EMT theory was founded in the 1980s during the development of electromagnetic pulse hardening studies (see [11]). The current formalism of the EMT is developed by Baum (see $[12,13])$. The EMT is often used to deal with a complex electromagnetic coupling problem.

To analyze the interaction processes for the electromagnetic coupling by the EMT, we need to establish the topological diagram and the topological network for the system configuration. Firstly, we consider a rectangular cavity with a slot aperture illuminated by a harmonic plane wave, see Figure 1. This field is described by angles of incidence $\psi$ and $\phi$, as well as a polarization angles $\alpha$, which defines the $E$-field vector direction relative to the vertical plane of incidence. Figure 2 shows the topological shielding diagram associated with 


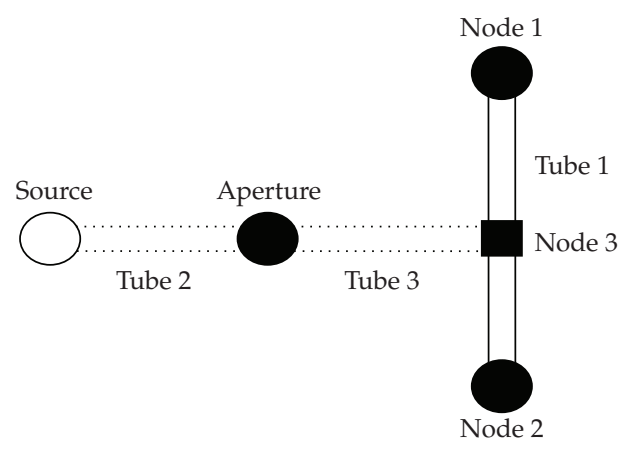

Figure 3: Topological network corresponding to the topological shielding diagram of Figure 2.

Figure 1. $V_{i, j}$ is a notation used for the different volumes. " $i$ " indicates the hierarchical order of the volume, " $j$ " indicates a number in the volume defined by $i . S_{i, j ; k, l}$ is a notation of a surface separating volumes $V_{i, j}$ and $V_{k, l}$. Figure 3 shows the topological network. Tube 1 is the transmission line tube; Tube 2 is the EM field propagation tube; Tube 3 is the EM field coupling tube. Based on the good shielding approximation theory (see [4]), the effect of the transmission line on the aperture can be neglected.

\subsection{The Aperture Coupling Model}

We introduce an equivalent magnetic current on the aperture as

$$
\bar{M}=M_{x} \widehat{x}+M_{y} \widehat{y},
$$

where

$$
\begin{aligned}
& M_{x}(x, y)=\sum_{p=1}^{P-1} \sum_{q=1}^{Q} M_{x p q} T_{p}\left(x-x_{a}\right) P_{q}\left(y-y_{b}\right), \\
& M_{y}(x, y)=\sum_{p=1}^{P} \sum_{q=1}^{Q-1} M_{y p q} P_{p}\left(x-x_{a}\right) T_{q}\left(y-y_{b}\right),
\end{aligned}
$$

in which $\left(x_{a}, y_{b}\right)$ are the coordinates of the lower left hand corner of the slot, and $T_{S}(t)$ and $P_{S}(t)$ are triangular and pulse functions defined by

$$
T_{p}(t)= \begin{cases}\frac{t-(p-1) \Delta t}{\Delta t}, & (p-1) \Delta t \leq t \leq p \Delta t \\ \frac{(p+1) \Delta t-t}{\Delta t}, & p \Delta t \leq t \leq(p+1) \Delta t \\ 0, & |t-p \Delta t| \geq \Delta t\end{cases}
$$


for $p=1,2, \ldots, P-1$, and

$$
P_{q}(t)= \begin{cases}1, & (q-1) \Delta t \leq t \leq q \Delta t \\ 0, & \text { otherwise, }\end{cases}
$$

for $q=1,2, \ldots, Q$.

To solve the unknown current $\bar{M}$, we enforce the continuity of the tangential magnetic field across the aperture. Then, we have

$$
\widehat{z} \times\left[\bar{H}^{a}(\bar{M})+\bar{H}^{i}\right]=\widehat{z} \times \bar{H}^{b}(\bar{M}), \quad z=0,
$$

where $\bar{H}^{a}(\bar{M})$ refers to the exterior scattered magnetic field, and $\bar{H}^{b}(\bar{M})$ referes to a corresponding magnetic field interior to the cavity.

The exterior scattered field can be expressed as the radiation caused by the equivalent magnetic current $\bar{M}$ (see [9]), say,

$$
\bar{H}^{a}(\bar{M})=-j k_{0} Y_{0} \int_{S} 2 \bar{M}\left(\bar{r}^{\prime}\right) \cdot \overline{\bar{\Gamma}}_{0}\left(\bar{r} ; \bar{r}^{\prime}\right) d s^{\prime},
$$

where $S$ denotes the surface of the aperture, $k_{0}$ is the wavenumber, $Y_{0}$ is the free space intrinsic admittance, and $\overline{\bar{\Gamma}}_{0}\left(\bar{r} ; \bar{r}^{\prime}\right)$ is the free space dyadic Green's function given by

$$
\begin{gathered}
\overline{\bar{\Gamma}}_{0}\left(\bar{r} ; \bar{r}^{\prime}\right)=\left(\overline{\bar{I}}+\frac{1}{k_{0}^{2}} \nabla \nabla\right) G_{0}\left(\bar{r} ; \bar{r}^{\prime}\right), \\
G_{0}\left(\bar{r} ; \bar{r}^{\prime}\right)=\frac{e^{-j k_{0}\left|\bar{r}-\bar{r}^{\prime}\right|}}{4 \pi\left|\bar{r}-\bar{r}^{\prime}\right|} \\
\left|\bar{r}-\bar{r}^{\prime}\right|=\sqrt{\left(x-x^{\prime}\right)^{2}+\left(y-y^{\prime}\right)^{2}},
\end{gathered}
$$

in which $\bar{r}$ and $\bar{r}^{\prime}$ represent the location of the field and source points, respectively, on the aperture.

Using the available dyadic, the Green function for the cavity, we can express the interior fields (see [6]) as follows:

$$
\begin{aligned}
& \bar{E}^{b}(\bar{M})=\int_{S} \nabla \times \overline{\bar{G}}_{H M} \cdot \bar{M} d s \\
& \bar{H}^{b}(\bar{M})=j \omega \varepsilon \int_{S} \overline{\bar{G}}_{H M} \cdot \bar{M} d s
\end{aligned}
$$


where the dyadic Green function is defined by

$$
\begin{aligned}
\overline{\bar{G}}_{H M}= & -\frac{1}{k_{0}^{2}} \widehat{z} \hat{z} \delta\left(\bar{R}-\bar{R}^{\prime}\right)-\sum_{m, n} \frac{2\left(2-\delta_{m n}\right)}{a b\left(k_{x m}^{2}+k_{y n}^{2}\right) k_{m n} \sin \left(k_{m n} c\right)} \\
& \cdot\left[\bar{M}_{o e}(z+c) \bar{M}_{o e}^{\prime}(0)+\bar{N}_{e o}(z+c) \bar{N}_{e o}^{\prime}(0)\right]
\end{aligned}
$$

where $\delta_{m n}$ ( $=1$ for $m=0$ or $n=0$, and 0 otherwise) denotes the Kronecker delta. The vector wave functions are given by

$$
\begin{gathered}
\bar{M}_{o e}(z)=\nabla \times\left(\sin \left(k_{x m} x\right) \sin \left(k_{y n} y\right) \cos \left(k_{z} z\right)\right), \\
\bar{N}_{e o}(z)=\frac{1}{k} \nabla \times \bar{M}_{o e}(z) .
\end{gathered}
$$

As usual, $k_{x m}=m \pi / a, k_{y n}=n \pi / b, k_{c}^{2}=k_{x m}^{2}+k_{y n}^{2}$ and

$$
k_{m n}= \begin{cases}-j \sqrt{k_{c}^{2}-k_{b}^{2}}, & k_{b}^{2}<k_{c}^{2}, \\ \sqrt{k_{b}^{2}-k_{c}^{2},} & k_{b}^{2}>k_{c}^{2} .\end{cases}
$$

Substituting (2.10) into (2.8) and (2.9), we obtain

$$
\begin{aligned}
\bar{E}^{b}(\bar{M})_{x}= & -\sum_{m, n} \frac{2 \varepsilon_{m}}{a b \sin \left(k_{m n} c\right)} I_{y}^{m n} \cos \left(k_{x m} x\right) \sin \left(k_{y n} y\right) \sin \left(k_{m n}(z+c)\right), \\
\bar{E}^{b}(\bar{M})_{y}= & \sum_{m, n} \frac{2 \varepsilon_{m}}{a b \sin \left(k_{m n} c\right)} I_{x}^{m n} \sin \left(k_{x m} x\right) \cos \left(k_{y n} y\right) \sin \left(k_{m n}(z+c)\right), \\
\bar{E}^{b}(\bar{M})_{z}= & -\sum_{m, n} \frac{2\left(2-\delta_{m n}\right)}{a b k_{m n} \sin \left(k_{m n} c\right)}\left[k_{y n} I_{x}^{m n}-k_{x m} I_{y}^{m n}\right] \\
& \cdot \sin \left(k_{x m} x\right) \sin \left(k_{y n} y\right) \cos \left(k_{m n}(z+c)\right), \\
H^{b}(\bar{M})_{x}= & -j Y_{b} k_{b} \sum_{m, n} \frac{2}{a b k_{m n} k_{c}^{2} \sin \left(k_{m n} c\right)} \\
& \cdot\left[\varepsilon_{n}\left(k_{y n}^{2}+\frac{k_{m n}^{2} k_{x m}^{2}}{k_{b}^{2}}\right) I_{x}^{m n}+\varepsilon_{m}\left(\frac{k_{m n}^{2} k_{x m} k_{y n}}{k_{b}^{2}}-k_{x m} k_{y n}\right) I_{y}^{m n}\right] \\
& \cdot \sin \left(k_{x m} x\right) \cos \left(k_{y n} y\right) \cos \left(k_{m n}(z+c)\right),
\end{aligned}
$$




$$
\begin{aligned}
H^{b}(\bar{M})_{y}= & -j Y_{b} k_{b} \sum_{m, n} \frac{2}{a b k_{m n} k_{c}^{2} \sin \left(k_{m n} c\right)} \\
& \cdot\left[\varepsilon_{n}\left(\frac{k_{m n}^{2} k_{x m} k_{y n}}{k_{b}^{2}}-k_{x m} k_{y n}\right) I_{x}^{m n}+\varepsilon_{m}\left(k_{x m}^{2}+\frac{k_{m n}^{2} k_{y n}^{2}}{k_{b}^{2}}\right) I_{y}^{m n}\right] \\
& \cdot \cos \left(k_{x m} x\right) \sin \left(k_{y n} y\right) \cos \left(k_{m n}(z+c)\right), \\
H^{b}(\bar{M})_{z}= & j Y_{b} k_{b} \sum_{m, n} \frac{2}{a b k_{m n} k_{c}^{2} \sin \left(k_{m n} c\right)}\left[\varepsilon_{n} \frac{k_{m n} k_{x m} k_{c}^{2}}{k_{b}^{2}} I_{x}^{m n}+\varepsilon_{m} \frac{k_{m n} k_{y n} k_{c}^{2}}{k_{b}^{2}} I_{y}^{m n}\right] \\
& \cdot \cos \left(k_{x m} x\right) \cos \left(k_{y n} y\right) \sin \left(k_{m n}(z+c)\right),
\end{aligned}
$$

where

$$
\begin{aligned}
I_{x}^{m n} & =\int_{S} M_{x}(x, y) \sin \left(k_{x m} x\right) \cos \left(k_{y n} y\right) d s, \\
I_{y}^{m n} & =\int_{S} M_{y}(x, y) \cos \left(k_{x m} x\right) \sin \left(k_{y n} y\right) d s .
\end{aligned}
$$

Now, we let $A_{m n}$ and $B_{m n}$ be defined by

$$
\begin{gathered}
A_{m n}=-\frac{2 j k_{b} Y_{b}}{k_{m n} a b \sin \left(k_{m n} c\right) k_{c}^{2}}\left[\varepsilon_{n} k_{y n} I_{x}^{m n}-\varepsilon_{m} k_{x m} I_{y}^{m n}\right], \\
B_{m n}=-\frac{2}{a b \sin \left(k_{m n} c\right) k_{c}^{2}}\left[\varepsilon_{n} k_{x m} I_{x}^{m n}+\varepsilon_{m} k_{y n} I_{y}^{m n}\right],
\end{gathered}
$$

where $Y_{b}=1 / Z_{b}=\left(1 / Z_{0}\right) \sqrt{\varepsilon_{b} / \mu_{b}}=\sqrt{\varepsilon / \mu}, k_{b}=k_{0} \sqrt{\mu_{b} \varepsilon_{b}}=\omega \sqrt{\mu \varepsilon}$,

$$
\varepsilon_{n}= \begin{cases}1, & n=0 \\ 2, & n>0\end{cases}
$$

The interior EM field can be expressed as follows:

$$
\begin{gathered}
E_{x}^{b}=\sum_{m, n}\left[\frac{j Z_{b}}{k_{b}} k_{x m} k_{m n} A_{m n}+k_{y n} B_{m n}\right] \cos \left(k_{x m} x\right) \sin \left(k_{y n} y\right) \sin \left(k_{m n}(z+c)\right), \\
E_{y}^{b}=\sum_{m, n}\left[\frac{j Z_{b}}{k_{b}} k_{x m} k_{m n} A_{m n}-k_{y n} B_{m n}\right] \sin \left(k_{x m} x\right) \cos \left(k_{y n} y\right) \sin \left(k_{m n}(z+c)\right), \\
E_{z}^{b}=-\sum_{m, n} \frac{j Z_{b} k_{c}^{2}}{k_{b}} A_{m n} \sin \left(k_{x m} x\right) \sin \left(k_{y n} y\right) \cos \left(k_{m n}(z+c)\right),
\end{gathered}
$$




$$
\begin{gathered}
H_{x}^{b}=\sum_{m, n}\left[k_{y n} A_{m n}+j \frac{Y_{b}}{k_{b}} k_{m n} k_{x m} B_{m n}\right] \sin \left(k_{x m} x\right) \cos \left(k_{y n} y\right) \cos \left(k_{m n}(z+c)\right), \\
H_{y}^{b}=\sum_{m, n}\left[-k_{x m} A_{m n}+j \frac{Y_{b}}{k_{b}} k_{m n} k_{y n} B_{m n}\right] \cos \left(k_{x m} x\right) \sin \left(k_{y n} y\right) \cos \left(k_{m n}(z+c)\right), \\
H_{z}^{b}=-\sum_{m, n} j \frac{Y_{b} k_{c}^{2}}{k_{b}} B_{m n} \cos \left(k_{x m} x\right) \cos \left(k_{y n} y\right) \sin \left(k_{m n}(z+c)\right) .
\end{gathered}
$$

In the formulas, $(m, n)$ are mode index numbers used for cavity modes and $(p, q)$ are integers used for aperture modes. We obtain the formulations of the interior EM field in which $M_{x p q}, M_{y p q}$ are constants to be determined.

In accordance with the method of weighted residuals (see [9]), the integral equation to be solved for $M_{x p q}, M_{y p q}$ is

$$
\int_{S} \widehat{z} \times\left[\bar{H}^{a}(\bar{M})+\bar{H}^{b}(\bar{M})\right] \cdot \bar{W} d s^{\prime}=-\int_{S} \widehat{z} \times \bar{H}^{i} \cdot \bar{W} d s^{\prime}, \quad z=0
$$

where $\bar{W}$ is a weighting function, $\bar{H}^{a}(\bar{M})$ and $\bar{H}^{b}(\bar{M})$ are given by (2.6) and (2.9). To discretize (2.23), the corresponding weighting function components are given by

$$
\begin{aligned}
& W_{x p^{\prime} q^{\prime}}(x, y)=P_{p^{\prime}}\left(x-x_{a}\right) T_{q^{\prime}}\left(y-y_{b}\right), \\
& W_{y p^{\prime} q^{\prime}}(x, y)=T_{p^{\prime}}\left(x-x_{a}\right) P_{q^{\prime}}\left(y-y_{b}\right) .
\end{aligned}
$$

Substitution of (2.2), (2.24) into (2.23) yields a system as follows:

$$
\left[Y^{a+b}\right][M]=\left[C^{\text {inc }}\right]
$$

The solution of this system (2.25) yields the coefficients $M_{x p q}, M_{y p q}$ in the magnetic current. There are some novel works for the system $(2.25)$ (see $[6,9])$.

\subsection{The Transmission Induction Model}

We consider the case where a lossless two-wire line is illuminated by a coupling EM field. In order to simplify the notation, let us place the two-wire transmission line in the $y=y_{0}$ plane which parallels to the $x$ axis as shown in Figure 4. We put the reference wire at $z=z_{0}$, and the other wire at $z=z_{0}+d$ so that there is distance $d$ between two wires. We assume that $d$ is greater than the wire radius $r_{a}\left(d \gg r_{a}\right) \cdot \rho_{i}=\left(Z_{i}-Z_{c}\right) /\left(Z_{i}+Z_{c}\right)(i=1,2)$ are the reflection coefficients at each node of the line. $L$ is the length of the line, $Z_{1}$ and $Z_{2}$ the load impedances at the ends $x=x_{0}$ and $x=x_{0}+L$, respectively, and $Z_{c}=120 \ln \left(d / r_{a}\right)$, the characteristic impedance of the line. The transmission line theory shows that the wave propagation constant $\gamma=j k_{0}$. 


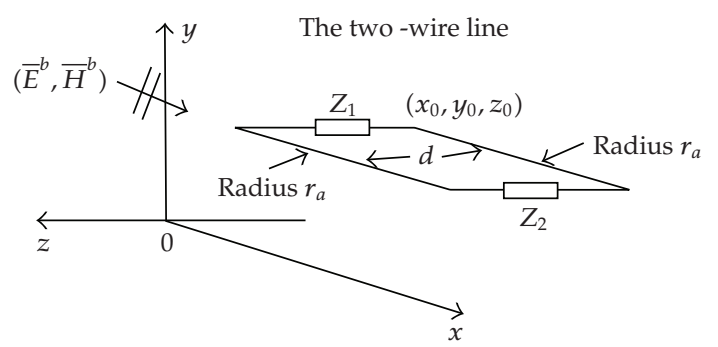

Figure 4: Isolated two-wire line excited by the interior field.

In the present paper, we only consider the transmission line mode current. The load currents and the total voltages at the loads can be expressed in matrix form as follows:

$$
\begin{aligned}
& {\left[\begin{array}{c}
I\left(x_{0}\right) \\
I\left(x_{0}+L\right)
\end{array}\right]=\frac{1}{Z_{c}}\left[\begin{array}{cc}
1-\rho_{1} & 0 \\
0 & 1-\rho_{2}
\end{array}\right]\left[\begin{array}{cc}
-\rho_{1} & e^{\gamma L} \\
e^{\gamma L} & -\rho_{2}
\end{array}\right]\left[\begin{array}{l}
S_{1} \\
S_{2}
\end{array}\right],} \\
& {\left[\begin{array}{c}
V\left(x_{0}\right) \\
V\left(x_{0}+L\right)
\end{array}\right]=\left[\begin{array}{cc}
1+\rho_{1} & 0 \\
0 & 1+\rho_{2}
\end{array}\right]\left[\begin{array}{cc}
-\rho_{1} & e^{\gamma L} \\
e^{\gamma L} & -\rho_{2}
\end{array}\right]\left[\begin{array}{l}
S_{1} \\
S_{2}
\end{array}\right] .}
\end{aligned}
$$

By the scattered voltage (Agrawal) formulation (see [14]), the source vector reads

$$
\left[\begin{array}{l}
S_{1} \\
S_{2}
\end{array}\right]=\frac{1}{2}\left[\begin{array}{c}
\int_{x_{0}}^{x_{0}+L} e^{\gamma x}\left[E_{x}^{b}\left(x, y_{0}, z_{0}+d\right)-E_{x}^{b}\left(x, y_{0}, z_{0}\right)\right] d x \\
+\int_{z_{0}}^{z_{0}+d}\left[E_{z}^{b}\left(x_{0}, y_{0}, z\right)-e^{\gamma L} E_{z}^{b}\left(x_{0}+L, y_{0}, z\right)\right] d z \\
-\left[\int_{x_{0}}^{x_{0}+L} e^{\gamma(L-x)}\left[E_{x}^{b}\left(x, y_{0}, z_{0}+d\right)-E_{x}^{b}\left(x, y_{0}, z_{0}\right)\right] d x\right. \\
\left.+\int_{z_{0}}^{z_{0}+d}\left[e^{\gamma L} E_{z}^{b}\left(x_{0}, y_{0}, z\right)-E_{z}^{b}\left(x_{0}+L, y_{0}, z\right)\right] d z\right]
\end{array}\right] .
$$

Substituting (2.17) and (2.19) into (2.27) leads to

$$
\begin{gathered}
S_{1}=\frac{1}{2} \sum_{m, n}\left\{\gamma e^{\gamma x_{0}} G_{m n}\left[e^{\gamma L} \cos \left(k_{x m}\left(x_{0}+L\right)\right)-\cos \left(k_{x m} x_{0}\right)\right]\right. \\
\left.+\left[e^{\gamma x_{0}} k_{x m} G_{m n}+H_{m n}\right]\left[e^{\gamma L} \sin \left(k_{x m}\left(x_{0}+L\right)\right)-\sin \left(k_{x m} x_{0}\right)\right]\right\}, \\
S_{2}=-\frac{1}{2} \sum_{m, n}\left\{\gamma e^{-\gamma x_{0}} G_{m n}\left[e^{\gamma L} \cos \left(k_{x m} x_{0}\right)-\cos \left(k_{x m}\left(x_{0}+L\right)\right)\right]\right. \\
\left.+\left[e^{-\gamma x_{0}} k_{x m} G_{m n}+H_{m n}\right]\left[\sin \left(k_{x m}\left(x_{0}+L\right)\right)-e^{\gamma L} \sin \left(k_{x m} x_{0}\right)\right]\right\},
\end{gathered}
$$




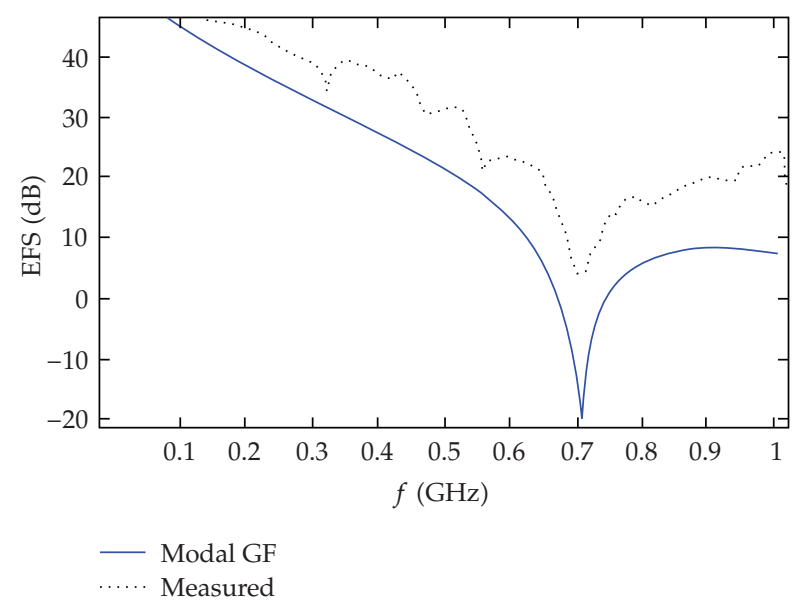

Figure 5: EFS for the empty cavity with an aperture by a plane wave.

with

$$
\begin{gathered}
G_{m n}=\frac{1}{\left(\gamma^{2}+k_{x m}^{2}\right)}\left[\frac{j Z_{b}}{k_{b}} k_{x m} k_{m n} A_{m n}+k_{y n} B_{m n}\right] C_{m n} \\
H_{m n}=\frac{j Z_{b} k_{c}^{2}}{k_{b} k_{m n}} A_{m n} C_{m n}
\end{gathered}
$$

where $C_{m n}=\sin \left(k_{y n} y_{0}\right)\left[\sin \left(k_{m n}\left(z_{0}+d+c\right)\right)-\sin \left(k_{m n}\left(z_{0}+c\right)\right)\right]$ and $A_{m n}, B_{m n}$ are given in (2.15). Inserting (2.28) into (2.26) gives the load responses for the plane wave excitation of the line.

\section{Simulations and Experimental Results}

We present some numerical examples to demonstrate the formulation given in the previous section. We consider the coupling in a cavity that has a two-wire transmission line along the $x$ direction in it, see Figure 1. The parameters are as follows: the polarization angle $\alpha=\pi / 2$, incident angles $\phi=0, \psi=0$, the amplitude of the incident $E$-field $\left|E^{i}\right|=1$, the cavity size $a=0.3 \mathrm{~m}, b=0.12 \mathrm{~m}, c=0.3 \mathrm{~m}$, the aperture size $l=0.1 \mathrm{~m}, w=0.005 \mathrm{~m}$, the observation point $(0.15 \mathrm{~m}, 0.06 \mathrm{~m},-0.15 \mathrm{~m})$, the wire radius $r_{a}=0.0003 \mathrm{~m}$, the wire length $L=0.15 \mathrm{~m}$, the wire separation distance $d=0.02 \mathrm{~m}$, the characteristic impedance $Z_{c} \approx 503 \Omega$, the load resistance $Z_{1}=Z_{2}=503 \Omega$ (matched loads).

Figure 5 shows the electric field shielding (EFS) factor given by EFS $=-20 \lg \left|\bar{E}^{b} / \bar{E}^{i}\right|$ in which $\bar{E}^{b}$ is the interior cavity field at an observation point and $\bar{E}^{i}$ is the incident field at the same location. The electric field shielding obtained by using the modal GF method is plotted in Figure 5 along with the experimental results from [15]. From it we can see that they agree very well. Moreover, one resonance peak can be observed in the frequency range from $0.1 \mathrm{GHz}$ to $1 \mathrm{GHz}$, which corresponds to the first resonance of the cavity. The first resonance of the cavity is $\operatorname{TE}_{101}=(c / 2) \sqrt{(1 / 0.3)^{2}+(0 / 0.12)^{2}+(1 / 0.3)^{2}}=707 \mathrm{MHz}$. 


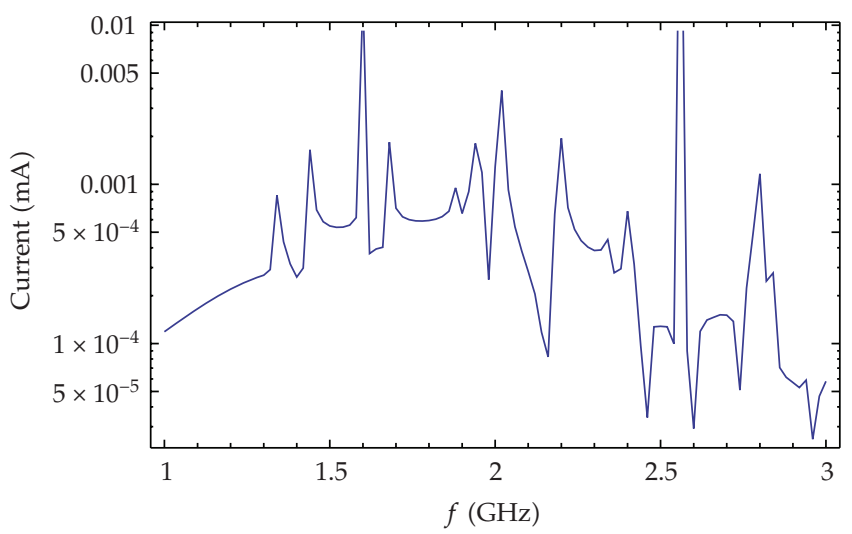

Figure 6: The induced current at Node 1 for a two-wire line in the cavity with an aperture.

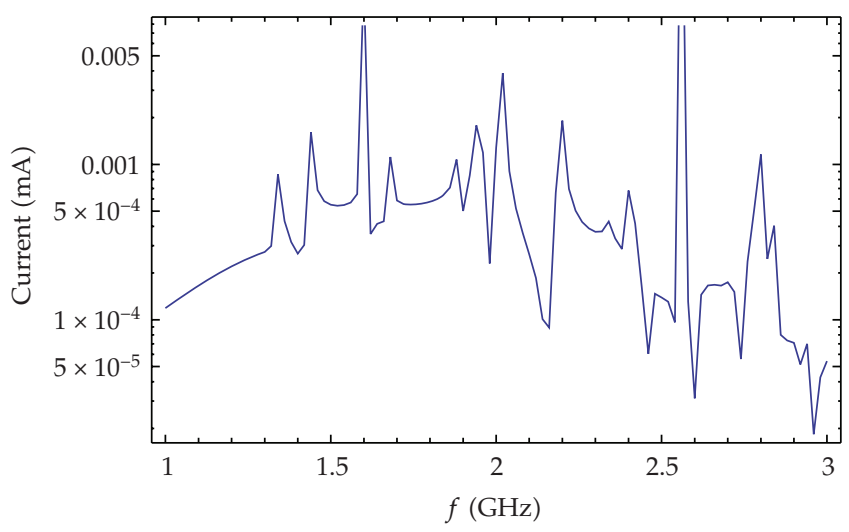

Figure 7: The induced current at Node 2 for a two-wire line in the cavity with an aperture.

The two-wire transmission line can be excited by the interior coupling electromagnetic field. Figures 6 and 7 show the results of the terminal response at Nodes 1 and 2, respectively. Four major induced current peaks at the terminal loads of the two-wire line are observed in the frequency range from $1 \mathrm{GHz}$ to $1.8 \mathrm{GHz}$, the second one of which is associated with the resonance of the aperture, while other three correspond to the first three resonant frequencies of the cavity. The first resonance of the cavity is $T E_{110}=1.35 \mathrm{GHz}$ and the next two higher resonances of the cavity are $T E_{210}=1.6 \mathrm{GHz}, T E_{211}=1.67 \mathrm{GHz}$. Moreover, these simulation results conclude that the currents and voltages can be derived from the interior coupling EM fields in the cavity with apertures.

\section{Conclusion}

We study the issue of field penetration through apertures and their coupling with a twowire transmission line. Especially, we use the Modal Green Function, the method of moment $(\mathrm{MoM})$, and the BLT equation to solve the load response of the two-wire transmission line in the cavity with a plane-wave excitation. It is observed from simulation results that the 
resonance of both the aperture and the cavity can occurs in the cavity. Therefore, PCB in the cavity can not be casually positioned and the circuit signal frequency should avoid these resonant frequencies which will cause strong induced current. Based on the EMT, our method can also be used for electromagnetic interaction problems on more complex systems. Hence, our work will aid shielding cavity design and electromagnetic interference (EMI) protection in the practical application.

\section{Acknowledgments}

The authors are grateful to the reviewers for their valuable comments and suggestions. This work was supported by China Postdoctoral Special Science Foundation (no. 200902662), the National Natural Science Foundation of China (no. 10871231), and Preresearch Foundation of Weapon and Equipment (no. 9140A31020609KG0170).

\section{References}

[1] F. C. Yang and C. E. Baum, "Use of Matrix Norms of Interaction Supermatrix Blocksfor Specifying Electromagnetic Performance of Subshields," Interaction Notes, 427, 1983.

[2] P. Kirawanich, R. Gunda, N. S. Kranthi, J. C. Kroenung, and N. E. Islam, "Methodology for interference analysis using electromagnetic topology techniques," Applied Physics Letters, vol. 84, no. 15, pp. 2949-2951, 2004.

[3] J. E. Nanevicz, E. F. Vance, W. Radasky, M. A. Uman, G. K. Soper, and J. M. Pierre, “EMP susceptibility insights from aircraft exposure to lightning," IEEE Transactions on Electromagnetic Compatibility, vol. 30, no. 4, pp. 463-472, 1988.

[4] M. Li, J. L. Drewniak, S. Radu, J. Nuebel, T. H. Hubing, R. E. DuBroff, and T. P. Van Doren, “An EMI estimate for shielding-enclosure evaluation," IEEE Transactions on Electromagnetic Compatibility, vol. 43, no. 3, pp. 295-304, 2001.

[5] B. A. Lail and S. P. Castillo, "Coupling through narrow slot apertures to thin-wire structures," IEEE Transactions on Electromagnetic Compatibility, vol. 42, no. 3, pp. 276-283, 2000.

[6] T. Yang, Coupling onto radio frequency components enclosed within canonical structures, Ph.D. dissertation, University of Michigan, 2006.

[7] D. W. P. Thomas, A. C. Denton, T. Konefal, T. Benson, C. Christopoulos, J. F. Dawson, A. Marvin, S. J. Porter, and P. Sewell, "Model of the electromagnetic fields inside a cuboidal enclosure populated with conducting planes or printed circuit boards," IEEE Transactions on Electromagnetic Compatibility, vol. 43, no. 2, pp. 161-169, 2001.

[8] R. Azaro, S. Caorsi, M. Donelli, and G. L. Gragnani, "A circuital approach to evaluating the electromagnetic field on rectangular apertures backed by rectangular cavities," IEEE Transactions on Microwave Theory and Techniques, vol. 50, no. 10, pp. 2259-2266, 2002.

[9] K. Barkeshli and J. L. Volakis, "Electromagnetic scattering from an aperture formed by arectangular cavity recessed in a ground plane," Journal of Electromagnetic Waves and Application, vol. 5, pp. 715-734, 1991.

[10] J.-M. Jin and J. L. Volakis, “TM scattering by an inhomogeneously filled aperture in a thick conducting plane," IEE Proceedings H, vol. 137, no. 3, pp. 153-159, 1990.

[11] J.-P. Parmantier, "Numerical coupling models for complex systems and results," IEEE Transactions on Electromagnetic Compatibility, vol. 46, no. 3, pp. 359-367, 2004.

[12] C. E. Baum, "The theory of the electromagnetic interference and reference data," Interaction Notes, 478, December 1989.

[13] C. E. Baum, Modern Radio Science, Oxford University Press, London, UK, 1990.

[14] F. M. Tesche, M. V. Ianoz, and T. Karlsson, EMC: Analysis Methods and Computational Models, John Wiley and Sons, New York, NY, USA, 1997.

[15] M. D. Deshpande, “Electromagnetic Field Penetration Studies," NASA Technical Paper, June 2000. 


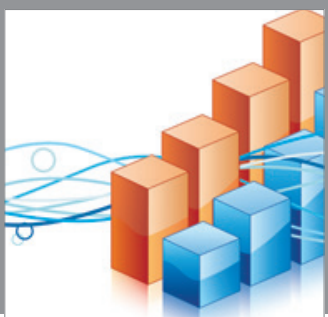

Advances in

Operations Research

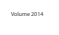

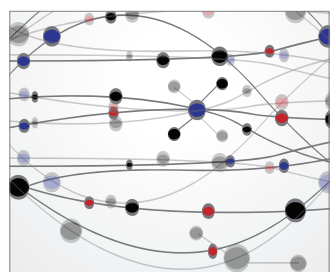

\section{The Scientific} World Journal
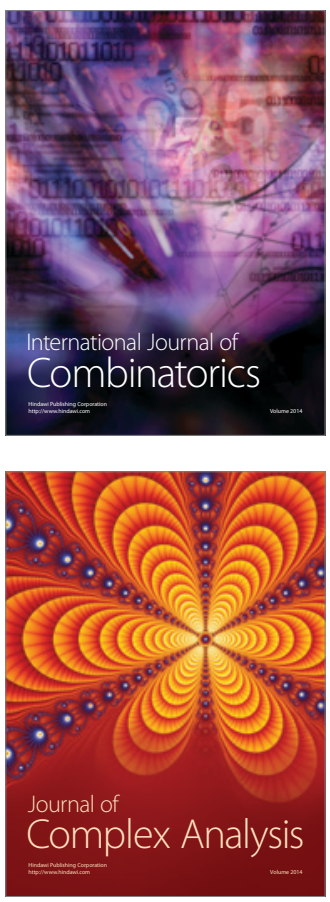

International Journal of

Mathematics and

Mathematical

Sciences
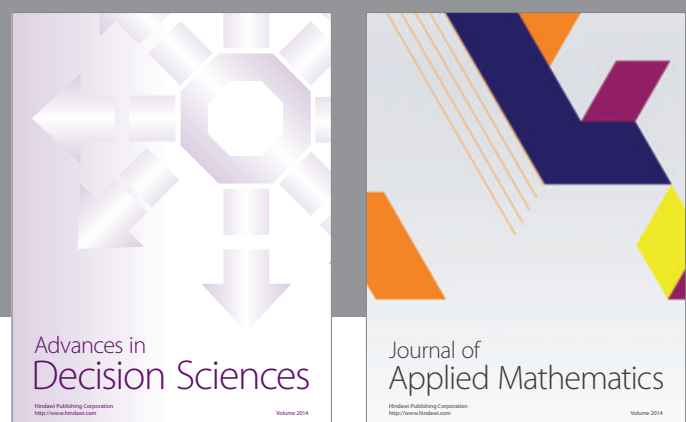

Journal of

Applied Mathematics
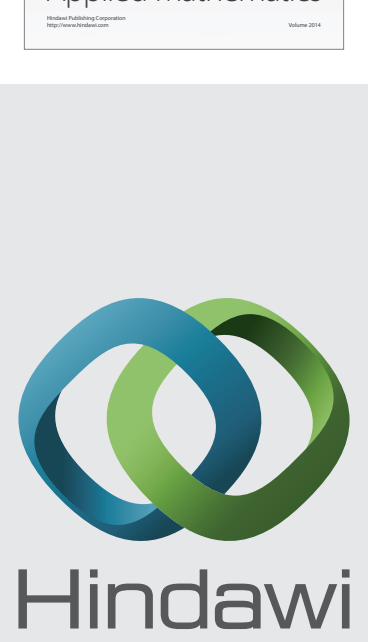

Submit your manuscripts at http://www.hindawi.com
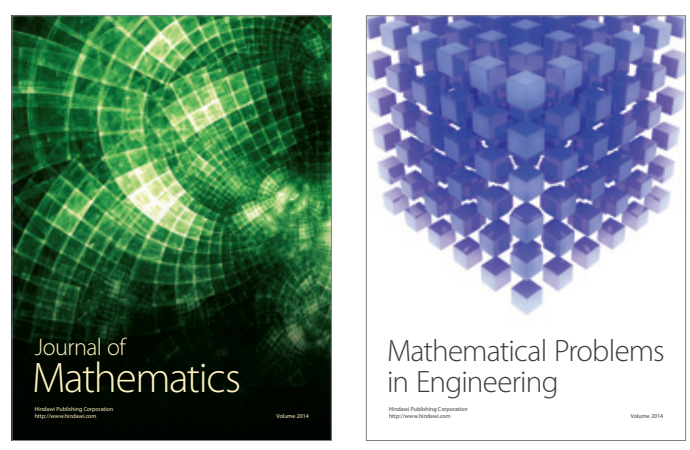

Mathematical Problems in Engineering
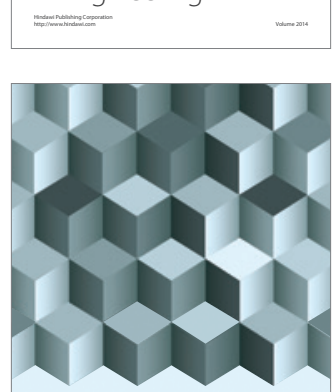

Journal of

Function Spaces
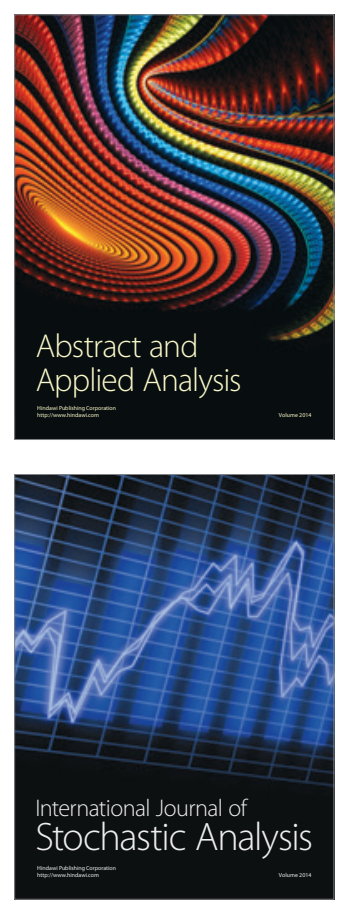

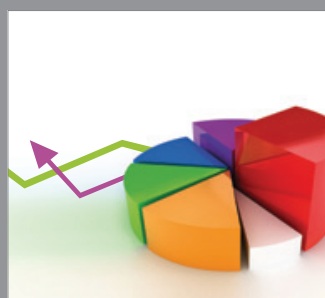

ournal of

Probability and Statistics

Promensencen
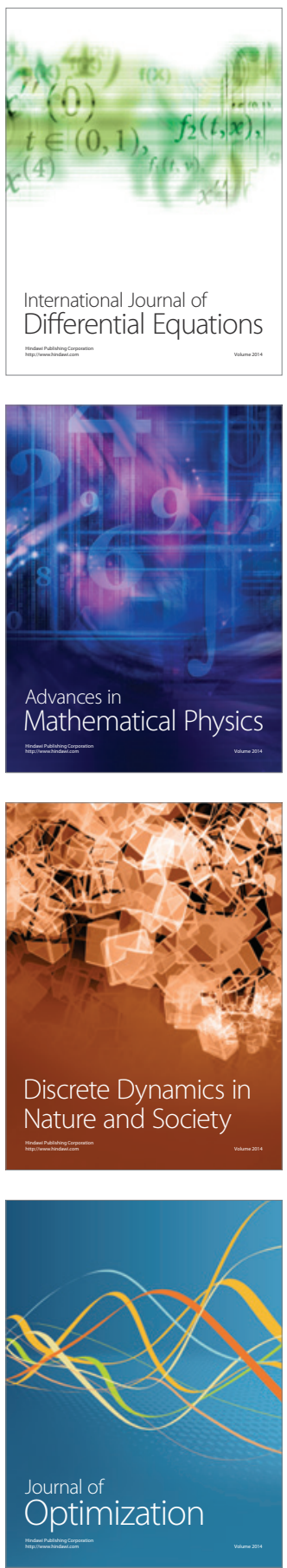